\title{
Density in the space of topological measures
}

by

S. V. Butler (Urbana-Champaign, IL)

\begin{abstract}
Topological measures (formerly "quasi-measures") are set functions that generalize measures and correspond to certain non-linear functionals on the space of continuous functions. The goal of this paper is to consider relationships between various families of topological measures on a given space. In particular, we prove density theorems involving classes of simple, representable, extreme topological measures and measures, hence giving a way of approximating various topological measures by members of different classes.
\end{abstract}

1. Introduction. Let $X$ be a compact Hausdorff space. Let $\mathcal{C}(X)$ (respectively $\mathcal{O}(X))$ denote the collection of closed (respectively open) subsets of $X$, and $\mathcal{A}(X)=\mathcal{C}(X) \cup \mathcal{O}(X)$. A topological measure on $X$ is a function $\mu: \mathcal{A}(X) \rightarrow \mathbb{R}^{+}$such that:

(i) $\mu\left(\bigsqcup_{i=1}^{n} A_{i}\right)=\sum_{i=1}^{n} \mu\left(A_{i}\right)\left(\sqcup\right.$ indicates disjoint union, and all $A_{i}$ and $\bigsqcup_{i=1}^{n} A_{i}$ are assumed to be in $\left.\mathcal{A}(X)\right)$;

(ii) $\mu(U)=\sup \{\mu(C): C \subseteq U, C \in \mathcal{C}(X)\}$ for all $U \in \mathcal{O}(X)$.

From (i) and (ii) it also follows that topological measures are monotone. Topological measures are not only finitely, but also countably additive: if $A=\bigsqcup_{i=1}^{\infty} A_{i}$, where $A, A_{i} \in \mathcal{A}(X)(i=1,2, \ldots)$, then $\mu(A)=\sum_{i=1}^{\infty} \mu\left(A_{i}\right)$ (see [15]). While topological measures resemble Borel measures, they in general need not be subadditive. Therefore, the class of topological measures is a larger collection of set functions on $\mathcal{A}(X)$ than the class of measures. Later we will show that there are situations when measures are nowhere dense in the space of all topological measures on $X$. A positive topological measure has a (necessarily unique) extension to a regular Borel measure on $X$ if and only if for any open sets $U$ and $V$ we have $\mu(U \cup V) \leq \mu(U)+\mu(V)$ (see [19]), while for a signed topological measure the condition for extension to a measure is $\mu(U)+\mu(V)=\mu(U \cup V)+\mu(U \cap V)$ (see [11]).

2000 Mathematics Subject Classification: 28C15, 41A65. 
REMARK 1.1. 1. Topological measures correspond to quasi-linear functionals, i.e. functionals that are only assumed to be linear on singly generated subalgebras in $C(X)$. The singly generated subalgebra generated by a continuous function $f$ is the smallest subalgebra of $C(X)$ that contains $f$ and constants. The Aarnes Representation Theorem (see [2]) enables one to restate the results for topological measures as results for quasi-linear functionals.

2. In this paper we assume that $X$ is compact Hausdorff. It is possible to consider the theory of topological measures in a more general setting, for example, taking a locally compact or a completely regular space. See [5], [8].

The paper is organized as follows. Section 2 provides some examples of topological measures that are not measures, and two basic construction techniques. The combination of these techniques is used in the proof of later results, while examples are linked to various families of topological measures discussed in Sections 3 and 4. In Section 3 we discuss the relationship between measures and topological measures. For example, if $X$ is an $n$-manifold with boundary, $n \geq 2$, the family of measures is nowhere dense in the set of topological measures. The known results included in this section give the reader an up-to-date picture of connections between measures and topological measures, and are also necessary for the results of the last section. The majority of new results are concentrated in Section 4, where we prove density theorems involving classes of extreme, representable topological measures and measures.

2. Examples and basic techniques. We will outline two important techniques for constructing topological measures. One uses $q$-functions, and the other uses solid sets. A set is solid if both the set and its complement are connected. To use the powerful technique of solid sets (developed by J. Aarnes in [4]) we also assume that $X$ is connected and locally connected.

For simplicity, in this paper we shall also assume that $X$ has a certain topological characteristic, genus $g=0$ (see [4] for details). One way to describe the " $g=0$ " condition is the following: if the union of two open solid sets in $X$ is the whole space, their intersection must be connected. (See [13].) Intuitively, $X$ does not have holes or loops. In the case where $X$ is locally path connected, $g=0$ if the fundamental group $\pi_{1}(X)$ is finite (in particular, if $X$ is simply connected), or, more generally, if the cohomology module $H^{1}(X)=0$ ([14], [17]). A compact, connected, locally connected, Hausdorff space will be called a $q$-space, and a $q$-space with genus 0 will be called a $q_{0}$-space. Examples of $q_{0}$-spaces include the unit ball in $\mathbb{R}^{n}, n \geq 2$, and the unit sphere in $\mathbb{R}^{n}, n \geq 3$. Notice that 1-dimensional $q_{0}$-spaces are dendrites, i.e. continua that are locally connected and contain no circles. 
Dendrites turn out to be very important in the theory of integration with respect to topological measures.

Let $\mathcal{C}_{\mathrm{s}}(X)$ (respectively $\mathcal{O}_{\mathrm{s}}(X)$ ) denote the family of closed (respectively open) solid sets in $X$. If $X$ is a $q_{0}$-space, a topological measure on $X$ is the unique extension of a solid set function, i.e. a set function $\mu: \mathcal{A}_{\mathrm{s}}(X)=$ $\mathcal{O}_{\mathrm{s}}(X) \cup \mathcal{C}_{\mathrm{s}}(X) \rightarrow \mathbb{R}^{+}$such that $1, \ldots, n$;

(A) $\sum_{i=1}^{n} \mu\left(C_{i}\right) \leq \mu(C)$ whenever $\bigsqcup_{i=1}^{n} C_{i} \subseteq C, C_{i}, C \in \mathcal{C}_{\mathrm{s}}(X), i=$

(B) $\mu(U)=\sup \left\{\mu(C): C \subseteq U, C \in \mathcal{C}_{\mathrm{s}}(X)\right\}$ for all $U \in \mathcal{O}_{\mathrm{s}}(X)$;

(C) $\mu(A)+\mu(X \backslash A)=\mu(X)$ for all $A \in \mathcal{A}(X)$.

We will also use $q$-functions, which were introduced in [7] and elaborated in $[9]$.

Definition 2.1. A function $f:[0,1] \rightarrow[0,1]$ is called a $q$-function if

1. $f$ is continuous from the right;

2. $f(0)=0, f(1)=1, f\left(x^{-}\right)+f(1-x)=1$ for $0<x \leq 1$;

3. $\sum_{i=1}^{n} x_{i}<1$ implies $\sum_{i=1}^{n} f\left(x_{i}\right) \leq f\left(\sum_{i=1}^{n} x_{i}\right)$.

Once we have a topological measure (or a solid set function) we may get a new one by applying a $q$-function. More precisely, we have the following theorem proved in [9]:

TheOrem 2.2. Let $X$ be a $q_{0}$-space, $\mu$ a topological measure on $X, f$ a $q$-function. Define a function $\nu$ on solid subsets of $X$ by: $\nu(C)=f(\mu(C))$ for all $C \in \mathcal{C}_{\mathrm{s}}(X)$, and $\nu(U)=1-\nu(X \backslash U)$ for all $U \in \mathcal{O}_{\mathrm{s}}(X)$. Then $\nu=f \circ \mu$ as defined above is a topological measure on $X$ if and only if $f$ is continuous on the split spectrum of $\mu$, i.e., on the set $\{\alpha \in(0,1)$ : there exist disjoint closed solid sets $C, C^{\prime} \in \mathcal{C}_{\mathrm{s}}(X)$ with $\left.\mu(C)=\alpha, \mu\left(C^{\prime}\right)=1-\alpha\right\}$.

In this paper we will use step functions of the form $f\left(I_{i}\right)=i / n$ where $I_{i}=[i /(n+1),(i+1) /(n+1))$ for $i=0, \ldots, n$ and $I_{n+1}=[n /(n+1), 1]$. We call such functions regular $(n+1)$-valued step $q$-functions.

On the space of all normalized topological measures on $X$, denoted by $\operatorname{TM}(X)$, we consider the weak* topology which can be described as generated by subbasic open sets:

$$
\widehat{U}(\alpha)=\{\mu: \mu(U)>\alpha\},
$$

where $U \in \mathcal{O}_{\mathrm{s}}(X), \alpha \in[0,1]$. The space $\operatorname{TM}(X)$ is a compact Hausdorff convex topological space. (See [1]; a more general result is in [16].)

Now we will give some examples of topological measures. In these examples topological measures are not subadditive, hence not measures. (This also implies that the corresponding functionals are only quasi-linear, but not linear.) 
Example 2.3. Let $X$ be the unit square and $B$ be the boundary of $X$. Fix a point $p$ in $X \backslash B$. Define $\mu$ on solid sets as follows: Let $\mu(A)=1$ if (i) $B \subset A$, or (ii) $p \in A$ and $A$ intersects $B$. Otherwise, we let $\mu(A)=0$. It is not very difficult to see that $\mu$ is a solid set function, and hence gives a topological measure on $X$. Notice that $\mu$ is not subadditive. Let $A_{1}$ be a closed solid set consisting of two adjacent sides of the boundary $B$, and $A_{2}$ be a closed solid set that is the other two adjacent sides of $B$. Let $A_{3}$ be the open solid set $X \backslash B$. Then we have: $X=A_{1} \cup A_{2} \cup A_{3}, \mu(X)=1$, but $\mu\left(A_{1}\right)+\mu\left(A_{2}\right)+\mu\left(A_{3}\right)=0$.

EXAmPLE 2.4. Let $X$ be the unit sphere, and let $P=\left\{p_{1}, \ldots, p_{n}\right\}$ be a set of $n$ distinct points in $X$, with $n$ an odd number. We define a solid set function $\mu$ on $X$ by letting $\mu(A)=1$ if $A \cap P$ contains a majority of points of $P$; otherwise let $\mu(A)=0$. The resulting topological measure $\mu$ is not subadditive, since it is not difficult to represent $X$ as a union of solid sets each containing less than half of the points.

The next example is a generalization of the previous one:

ExAmple 2.5. Let $X$ be the unit sphere and $P=\left\{p_{1}, \ldots, p_{2 n+1}\right\}$ be a subset of $X$. We use Theorem 2.2 to obtain a topological measure $\mu=f \circ \nu$ where $f$ is the regular $(n+1)$-valued step $q$-function and $\nu$ is the average of point masses at $p_{1}, \ldots, p_{2 n+1}$. If $A \in \mathcal{A}_{\mathrm{s}}(X)$ and $A$ contains $2 k$ or $2 k+1$ points from the set $P$ then $\mu(A)=k / n$. Again, it is not difficult to check that $\mu$ is not subadditive.

We already mentioned that measures constitute a subfamily in the space of topological measures. The examples above will enable us to introduce a few other families of topological measures in the space $\operatorname{TM}(X)$.

One goal of this paper is to show how members of one family of topological measures can be approximated by elements of another family. We say that $Y$ is dense in $Z$ if $Z$ is contained in the closure of $Y$. The set $Z$ may or may not be a subset of $Y$.

\section{Measures and simple topological measures}

Definition 3.1. A topological measure is simple if it only assumes values 0 and 1 .

Note that the only simple measures are point masses. Examples 2.3 and 2.4 show that there are simple topological measures that are not measures.

Definition 3.2. A topological measure $\mu$ on $X$ is finitely defined if there is a finite subset $P$ of $X$ such that $\sum_{i=1}^{n} \mu\left(A_{i}\right) \leq \mu(A)$ whenever $\bigsqcup_{i=1}^{n}\left(A_{i} \cap P\right)$ $\subseteq A \cap P$ and $A, A_{1}, \ldots, A_{n} \in \mathcal{A}_{\mathrm{s}}(X)$. 
Topological measures in Examples 2.4 and 2.5 are finitely defined, whereas the topological measure in Example 2.3 is not. Later on we will use the following theorem, first proved by F. Knudsen in [17].

TheOREM 3.3. Any simple topological measure on a $q_{0}$-space can be approximated by finitely defined simple topological measures.

For the sake of completeness we will give a short proof of the theorem, based on the following definition and lemma.

Definition 3.4. A family of sets $\left\{A_{k}\right\}_{k=1}^{n}$ is called linked if $A_{i} \cap A_{j} \neq \emptyset$ for any $i, j \in\{1, \ldots, n\}$.

Lemma 3.5. Let $\left\{U_{k}\right\}_{k=1}^{n}$ (where $\left.n \geq 1\right)$ be a linked family of open solid subsets of a $q_{0}$-space $X$. Then there exists a simple finitely defined topological measure $\mu$ such that $\mu\left(U_{k}\right)=1$ for all $k=1, \ldots, n$.

Proof. The proof is by induction on $n$.

- $n=1$. We may choose $\mu$ to be a point mass $\delta_{x}$ for any $x \in U_{1}$.

- $n=2$. Take $\mu=\delta_{x}$ for any $x \in U_{1} \cap U_{2}$.

- Assuming the result for $n$, we will prove the statement in the case $n+1$, where $n \geq 2$. Let $\left\{U_{k}\right\}_{k=1}^{n+1}$ be a linked family of open solid sets. For each $j \in\{1, \ldots, n\}$ the set $\left\{U_{k}: k \neq j\right\}$ is a linked family of $n$ sets. By assumption there exists a $\{0,1\}$-valued finitely defined topological measure $\mu_{j}$ such that $\mu_{j}\left(U_{k}\right)=1$ for all $k \neq j$. Consider a topological measure $\nu=\left(\mu_{1}+\ldots\right.$ $\left.\ldots+\mu_{n+1}\right) /(n+1)$ if $n$ is even and $\nu=\left(\mu_{1}+\mu_{1}+\mu_{2}+\ldots+\mu_{n+1}\right) /(n+2)$ if $n$ is odd. Then $\nu$ is finitely defined and for all $j \in\{1, \ldots, n+1\}, \nu\left(U_{j}\right)>$ $1 / 2$. If $q$ is the regular 2-valued $q$-function, then by Theorem 2.2, $\mu=q \circ \nu$ is a simple finitely defined topological measure, and $\mu\left(U_{j}\right)=1$ for all $j=$ $1, \ldots, n+1$.

Proof of Theorem 3.3. Let $\mu$ be a simple topological measure in a basic open set $W=\bigcap_{i=1}^{n} \widehat{U}_{i}\left(\alpha_{i}\right)$ in the space $\operatorname{TM}(X)$, where each $\alpha_{i} \in(0,1)$, and $U_{i}$ is a solid subset of $X$. Then $\mu\left(U_{i}\right)>\alpha_{i}$, i.e. $\mu\left(U_{i}\right)=1$ for $i=1, \ldots, n$. This implies that the family $\left\{U_{i}\right\}_{i=1}^{n}$ is a linked family of open solid sets. (Otherwise, there would be disjoint sets $U_{i}, U_{j}$, and then $\mu(X) \geq \mu\left(U_{i} \sqcup U_{j}\right)$ $=\mu\left(U_{i}\right)+\mu\left(U_{j}\right)=2$, which is a contradiction.) By Lemma 3.5 there exists a finitely defined simple topological measure which belongs to $W$.

In view of Theorem 3.3 one may ask whether any topological measure can be approximated by finitely defined ones. The affirmative answer to this question is given in [6].

TheOREM 3.6. Finitely defined topological measures are dense in the collection of all topological measures on a $q_{0}$-space. 
The set of measures on $X$ is a closed convex subset of $\operatorname{TM}(X)$. This statement is easy to check using the description of the topology on $\operatorname{TM}(X)$ given by quasi-linear functionals: $\mu_{\alpha} \rightarrow \mu$ if and only if for the corresponding functionals we have $\varrho_{\alpha}(f) \rightarrow \varrho(f)$ for all $f \in C(X)$. The relationship between topological measures and quasi-linear functionals is given in the Aarnes Representation Theorem (see [2]). If $\mu$ is a measure, then the corresponding functional is linear, and the relationship between the two is the standard one, given by the Riesz Representation Theorem.

Theorem 3.7. If $X$ is a compact n-manifold with boundary, $n \geq 2$, then the family of measures is nowhere dense in the space $\operatorname{TM}(X)$.

This theorem holds for an arbitrary genus of $X$. In this paper we are interested in the situation $g=0$, which we discussed above. For the general definition of genus the reader is referred to [4].

Proof. Let $m$ be a measure in $W=\bigcap_{j=1}^{l} \widehat{U}_{j}\left(\alpha_{j}\right)$, a non-empty basic open set in the space $\operatorname{TM}(X)$. In particular, $m\left(U_{j}\right)>\alpha_{j}$ for $j=1, \ldots, l$. By the Krein-Milman Theorem, $m$ is approximated by a convex combination of extreme points of $\operatorname{TM}(X)$ which are measures, i.e. by a convex combination of point masses. We may assume that

$$
m=\sum_{i=1}^{n} r_{i} \delta_{i}
$$

where $r_{i}$ are rational numbers and $\delta_{i}$ are point masses at points $p_{i}$. We may rewrite $m$ as

$$
m=\frac{q_{1} \delta_{1}+\ldots+q_{n} \delta_{n}}{q}
$$

where $q=q_{1}+\ldots+q_{n}$. Let $V_{1}, \ldots, V_{n}$ be $q_{0}$-neighborhoods around the points $p_{1}, \ldots, p_{n}$ so that each $V_{i}$ is in the same $U_{j}$ as the point $p_{i}$. Inside each $V_{i}$ we may construct a topological measure $\mu_{i}$ which is not a measure. (For instance, a topological measure as in Examples 2.3 or 2.4 will do.) Then

$$
\mu=\frac{q_{1} \mu_{1}+\ldots+q_{n} \mu_{n}}{q}
$$

is a topological measure which is not a measure, since its restriction to each $U_{j}$ is not a measure. We also have

$$
\mu\left(U_{j}\right) \geq\left\{\sum q_{i} \mu\left(V_{i}\right) / q: V_{i} \subset U_{j}\right\}=\left\{\sum q_{i} / q: p_{i} \in U_{j}\right\}=m\left(U_{j}\right)>\alpha_{j},
$$

in other words, a topological measure $\mu$ is in $W$.

From the previous proof it is easy to see that measures are nowhere dense in the collection of topological measures on $X$ if each point of $X$ has a contractible neighborhood of $\operatorname{dim} \geq 2$. The dimension condition is the crucial one. Work on the theory of topological measures and dimension 
theory initiated by R. Wheeler, and continued by D. Shakmatov, D. Fremlin, and D. Grubb, describes a very different relationship between measures and topological measures on a space of dimension not greater than 1. See [19], [18], [10] or [12] (a more general result).

Theorem 3.8. If $\operatorname{dim}(X) \leq 1$ then any topological measure is a measure, i.e. the family of measures is $\operatorname{TM}(X)$.

\section{Representable and extreme topological measures}

Definition 4.1. We say a topological measure is representable if it belongs to the closed convex hull of the set of simple topological measures.

Representable topological measures (and corresponding functionals that are also called representable) were introduced by J. Aarnes in [3]. The next theorem we state is due to J. Aarnes as well.

Let $X^{*}$ denote the set of all simple topological measures on $X$, and $\mathcal{P}\left(X^{*}\right)$ be the collection of all subsets of $X^{*}$. Define a map $\Psi^{*}: \mathcal{A}(X) \rightarrow$ $\mathcal{P}\left(X^{*}\right)$ by $\Psi^{*}(A)=\left\{\mu \in X^{*}: \mu(A)=1\right\}$.

TheOREM 4.2. For each positive, regular Borel measure $m$ on $X^{*}$ with $m\left(X^{*}\right)=1$ the set function $\mu$ defined by

$$
\mu(A)=\left(m \circ \Psi^{*}\right)(A) \quad \text { for } A \in \mathcal{A}(X)
$$

is a representable topological measure on $X$. Conversely, for each representable topological measure $\mu$ on $X$ there is a positive, regular Borel measure $m$ on $X^{*}$ with $m\left(X^{*}\right)=1$ satisfying condition $(*)$.

The proof of this theorem and other facts about representable topological measures and quasi-linear functionals are in [3].

Any measure is a representable topological measure. However, we will show below that representable topological measures do not give the whole space $\operatorname{TM}(X)$.

To discuss extreme topological measures we will use the notion of a solidly chainable space. Let $\mathcal{F}$ be a finite collection of subsets of $X$. We denote the number of elements of $\mathcal{F}$ by $|\mathcal{F}|$. For a subset $C \subseteq X$ let $n(C)$ be the number of sets from $\mathcal{F}$ that are subsets of $C$. Thus $n(C) \leq|\mathcal{F}|$ for any set $C$.

Definition 4.3. A finite family $\mathcal{F}=\left\{F_{1}, \ldots, F_{m}\right\}$ of sets is chained if $F_{i} \cap F_{j}=\emptyset$ if and only if $1<|i-j|<m-1$.

Definition 4.4. A space $X$ is solidly chainable with respect to a finite subset $P$ if for a chained family $\mathcal{F}=\left\{F_{1}, \ldots, F_{t}\right\}$ of subsets of $P$ and a closed solid set $C_{1}$ in $X$, with $n\left(C_{1}\right) \geq 1$, which contains sets $F_{1}, \ldots, F_{n\left(C_{1}\right)}$ and does not intersect $(\bigcup \mathcal{F}) \backslash\left(F_{1} \cup \ldots \cup F_{n\left(C_{1}\right)}\right)$, there exists a chained family 
$\left\{C_{1}, \ldots, C_{m}\right\}$ of closed solid sets such that $n\left(C_{i}\right)=1$ for $i=2, \ldots, m$, where $m=t-n\left(C_{1}\right)+1$.

$X$ is solidly chainable if it is solidly chainable with respect to any finite subset.

Notice that since $\mathcal{F}$ is a chained family, in Definition 4.4 the assumption that $C_{1}$ contains sets $F_{1}, \ldots, F_{n\left(C_{1}\right)}$ may be replaced by the assumption that $C_{1}$ contains $n\left(C_{1}\right)$ consecutive members of $\mathcal{F}$.

EXAMPLE 4.5. The unit sphere $S^{k}$ is solidly chainable for $k \geq 2$. In fact, given $C_{1}$ with $n\left(C_{1}\right)=1$, one may choose closed solid arcs $C_{2}, \ldots, C_{m}$ as follows. Beginning with $t>3$ (the only interesting case), let $C_{2}$ be an arc such that $C_{2} \cap P=F_{2}$. For $i=3, \ldots, m-1$ choose $C_{i}$ to be an arc through the points of $F_{i}$ such that $C_{i} \cap\left(C_{1} \cup \ldots \cup C_{i-1}\right)$ is an arc through the points of $F_{i} \cap F_{i-1}$. Let $C_{m}$ be an arc through the points of $F_{m}$ such that $C_{m} \cap\left(C_{2} \cup \ldots \cup C_{m-1}\right)$ is an arc through the points of $F_{m} \cap F_{m-1}$.

Similarly, the unit ball $B^{k}$ is solidly chainable with respect to any finite set $F$ that does not intersect the boundary of the ball for $k=1,2$. For $k \geq 3$ the unit ball $B^{k}$ is solidly chainable with respect to any finite set.

Notice that if $X$ is a unit square in $\mathbb{R}^{2}$ and $P$ is a finite subset of $X$ that has points on the boundary of the square, then $X$ may not be solidly chainable with respect to $P$. Suppose $P=\left\{p_{1}, p_{2}, p_{3}, p_{4}\right\}$ where each segment of the boundary contains exactly one of these points, and points $p_{1}, p_{2}$ are on the opposite segments. Then $\mathcal{F}=\left\{\left\{p_{1}, p_{2}\right\},\left\{p_{2}, p_{3}\right\},\left\{p_{3}, p_{4}\right\},\left\{p_{4}, p_{1}\right\}\right\}$ is a chained family of subsets of $P$ with no corresponding chained family of closed solid sets. Any solid set in $X$ containing points $p_{1}, p_{2}$ cannot be disjoint from a solid set containing points $p_{3}, p_{4}$.

REMARK 4.6. Topological measures in all three examples in Section 2 are extreme points in the space $\operatorname{TM}(X)$. Notice that the only extreme measures are point masses. Theorems 4.7 (which is proved in a more general form in [9]) and 4.8 show that we have a large family of extreme topological measures.

THEOREM 4.7. Let $\mu_{1}, \ldots, \mu_{(n+1) l-1}$ (where $n, l$ are natural numbers) be simple topological measures on $X$ that are finitely defined by disjoint sets, $\mu$ be the average of $\mu_{1}, \ldots, \mu_{(n+1) l-1}$, and $q$ be the regular $(n+1)$-valued step $q$-function. If $X$ is a solidly chainable $q_{0}$-space then $\nu=q \circ \mu$ is a topological measure on $X$ that is extreme if and only if $n=1$ or $l \geq 2$.

This theorem shows that for any natural $n$ there are infinitely many extreme topological measures that assume values $0,1 / n, \ldots, 1$.

THEOREM 4.8. Extreme topological measures are dense in the set of representable topological measures on a solidly chainable $q_{0}$-space. 
Proof. To prove the theorem we need to show that if $\mu$ is a convex combination of simple topological measures that belongs to a basic open set $W$ of $\operatorname{TM}(X)$ then there exists an extreme topological measure $\nu$ which is also in $W$.

Let $\mu$ be a convex combination of simple topological measures which belongs to a basic open set $W=\bigcap_{i=1}^{l} \widehat{U}_{i}\left(\alpha_{i}\right)$ in the space $\operatorname{TM}(X)$, where each $U_{i}$ is a solid subset of $X$ and $\alpha_{i} \in(0,1)$. Whenever $\left\{U_{j}\right\}_{j \in J}$ is a disjoint subcollection of $\left\{U_{1}, \ldots, U_{l}\right\}$ we have $\sum_{j \in J} \alpha_{j}<1$, since otherwise we would have $\mu(X) \geq \sum_{j \in J} \mu\left(U_{j}\right)>\sum_{j \in J} \alpha_{j} \geq 1$. Choose $\beta_{i}$ such that $\alpha_{i}<\beta_{i}<\mu\left(U_{i}\right)$ for $i=1, \ldots, l$. In other words, $\mu \in W^{\prime} \subseteq W$, where $W^{\prime}=\bigcap_{i=1}^{l} \widehat{U}_{i}\left(\beta_{i}\right)$.

Suppose $\mu=\sum_{i=1}^{m} r_{i} \mu_{i}$ with $\sum_{i=1}^{m} r_{i}=1$. We may assume that all $r_{i}$ are rational. Using Theorem 3.3 we may also assume that all $\mu_{i}$ are finitely defined simple topological measures. Then we can write

$$
\mu=\frac{k_{1} \mu_{1}+\ldots+k_{m} \mu_{m}}{\sum_{i=1}^{m} k_{i}} .
$$

Multiplying the numerator and the denominator by an appropriate integer we may take the denominator to be as large a number as we wish. For a sufficiently large denominator, adding a point mass to one of the sets $U_{i}$ will give a new topological measure that is still in $W^{\prime}$. Hence we may assume that the denominator is an odd number.

If $\lambda$ is any finitely defined topological measure determined by the set $F_{1}$, we may replace $k \lambda$ with $k$ different topological measures $\lambda_{1}, \ldots, \lambda_{k}$ that are determined by disjoint finite sets. We just need to move the set $F_{1}$ that defines $\lambda$ a little bit to get disjoint "copies" of it, sets $F_{2}, \ldots, F_{k}$, so that $\left|F_{j} \cap U_{i}\right|=\left|F_{1} \cap U_{i}\right|$ for all $j=2, \ldots, k$ and all $i=1, \ldots, l$. Hence, we may now consider $\mu \in W^{\prime}$ of the form

$$
\mu=\frac{\mu_{1}+\ldots+\mu_{m}}{m},
$$

where $\mu_{i}$ are simple topological measures finitely defined by disjoint sets, and $m$ is as large an odd number as we wish.

Choose $m=2 n+1$ so that $1 /(n+1)<\min \left\{\beta_{i}-\alpha_{i}: i=1, \ldots, l\right\}$. Then for $i=1, \ldots, l$ there exist $j_{i}$ such that

$$
\alpha_{i} \leq \frac{j_{i}}{n+1}<\frac{j_{i}+1}{n+1}<\beta_{i}<\mu\left(U_{i}\right)
$$

Let $q$ be the regular $(n+1)$-valued step $q$-function, i.e. $q\left(\left[\frac{j}{n+1}, \frac{j+1}{n+1}\right)\right)=j / n$ for $j=0, \ldots, n-1$, and $q\left(\left[\frac{n}{n+1}, 1\right]\right)=1$. By Theorem 4.7 the topological measure $\nu=q \circ \mu$ is extreme. For $i=1, \ldots, l$ we have

$$
\nu\left(U_{i}\right)=q\left(\mu\left(U_{i}\right)\right)>j_{i} / n>j_{i} /(n+1) \geq \alpha_{i} .
$$

This means that the extreme topological measure $\nu$ is in $W$. 
Theorem 4.9. Let $X$ be a solidly chainable $q_{0}$-space. Then measures on $X$ are approximated by extreme topological measures.

Proof. This follows from Theorem 4.8 and the fact that any measure is a representable topological measure.

In the proof of the next theorem we will need the following result (see Lemma 2.22 in [9], and Lemma 3.3 in [4]):

Lemma 4.10. Let $X$ be a q-space. Let $K \subseteq U$, where $K \in \mathcal{C}(X)$ and $U \in \mathcal{O}_{\mathrm{s}}(X)$, or $K \in \mathcal{C}_{\mathrm{s}}(X)$ and $U \in \mathcal{O}(X)$. Then there exist $V \in \mathcal{O}_{\mathrm{s}}(X)$ and $C \in \mathcal{C}_{\mathrm{s}}(X)$ such that $K \subseteq V \subseteq C \subseteq U$.

Theorem 4.11. Suppose that $X$ is a solidly chainable $q_{0}$-space. For $n \geq 1$ let $q$ be the regular $(n+1)$-valued step $q$-function, and $\mu=\left(\mu_{1}+\right.$ $\left.\ldots+\mu_{2 n+1}\right) /(2 n+1)$, where $\mu_{1}, \ldots, \mu_{2 n+1}$ are simple topological measures on $X$ finitely defined by disjoint sets. Then the extreme topological measure $\nu=q \circ \mu$ is representable if and only if $n=1$.

Proof. $(\Rightarrow)$ If $n=1$ then the topological measure $\nu$ is simple, hence representable.

$(\Leftarrow)$ Let $n \geq 2$. By Theorem 4.7, $\nu$ is an extreme topological measure. We will show that $\nu$ is not representable. Choose a positive number $\alpha$ which satisfies the inequality

$$
\frac{1}{n+1}<\frac{2}{2 n+1}<\alpha<\frac{1}{n}=\frac{2}{2 n} .
$$

Then we also have:

$$
\begin{aligned}
& (n+1) \alpha>1, \quad \text { i.e. } \quad 1-n \alpha<\alpha, \\
& (n+1) \alpha>\frac{2(n+1)}{2 n+1}, \quad \text { i.e. } \frac{2(n+1)}{2 n+1}-n \alpha<\alpha .
\end{aligned}
$$

Now we will outline the construction of a basic open set $W$ in $\operatorname{TM}(X)$ of the form

$$
W=\bigcap_{i=1}^{2 n+1} \widehat{U}_{i}(\alpha),
$$

where $U_{i}$ are open solid sets in $X$ that are linked in a circular chain, i.e. $U_{i} \cap U_{j}=\emptyset$ if and only if $1<|i-j|<2 n$.

Suppose $P_{1}, \ldots, P_{2 n+1}$ are disjoint finite sets determining $\mu_{1}, \ldots, \mu_{2 n+1}$. Form a family of $2 n+1$ finite sets: $\mathcal{F}=\left\{\left\{P_{1} \sqcup P_{2}\right\}, \ldots,\left\{P_{2 n+1} \sqcup P_{1}\right\}\right\}$. By Lemma 4.10 there exists a solid set $C_{1}$ containing the set $P_{1} \sqcup P_{2}$. Since $X$ is solidly chainable, we may find solid sets $C_{2}, \ldots, C_{2 n+1}$ containing the sets $P_{2} \sqcup P_{3}, \ldots, P_{2 n+1} \sqcup P_{1}$ respectively, that are linked in a circular chain. Using Lemma 4.10 we may enlarge $C_{1}, \ldots, C_{2 n+1}$ to open solid sets 
$U_{1}, \ldots, U_{2 n+1}$ that form a circular chain and contain respectively the sets $P_{1} \sqcup P_{2}, \ldots, P_{2 n+1} \sqcup P_{1}$.

Notice that $\nu \in W$, because $\nu\left(U_{i}\right)=1 / n>\alpha$ for $i=1, \ldots, 2 n+1$. We will show that no convex combination of simple topological measures is in $W$.

Suppose the opposite. Let $\nu^{\prime}=\sum_{j=1}^{m} \lambda_{j} \nu_{j}$, where $\nu_{j}$ are simple topological measures and $\sum_{j=1}^{m} \lambda_{j}=1$, be in $W$. For each $i=1, \ldots, 2 n+1$ consider the set

$$
I_{i}=\left\{j \in\{1, \ldots, m\}: \nu_{j}\left(U_{i}\right)=1\right\} .
$$

Notice that

(a) $\nu^{\prime}\left(U_{i}\right)=\sum_{j \in I_{i}} \lambda_{j}$.

(b) $\alpha<\nu^{\prime}\left(U_{i}\right)<1-\alpha$ for $i=1, \ldots, 2 n+1$. (Since $n \geq 2$, for any $U_{i}$ we may find a disjoint set $U_{j}$. Then $\nu^{\prime}\left(U_{i}\right)+\nu^{\prime}\left(U_{j}\right) \leq 1$. Using the fact that $\nu^{\prime} \in W$ we get $\alpha<\nu^{\prime}\left(U_{i}\right) \leq 1-\nu^{\prime}\left(U_{j}\right)<1-\alpha$.)

(c) $U_{i} \cap U_{k}=\emptyset \Rightarrow I_{i} \cap I_{k}=\emptyset$. (If there exists $j \in I_{i} \cap I_{k}$, then $\nu_{j}(X) \geq$ $\nu_{j}\left(U_{i}\right)+\nu_{j}\left(U_{k}\right)=2$, which is a contradiction.)

Introduce sets $E_{i}=I_{i} \cap I_{i+1}$ for $i=1, \ldots, 2 n$ and $E_{2 n+1}=I_{2 n+1} \cap I_{1}$, and consider two cases.

CASE 1: There exists $i \in\{1, \ldots, 2 n+1\}$ such that $E_{i}=\emptyset$. Say, $E_{1}=\emptyset$, i.e. $I_{1} \cap I_{2}=\emptyset$. Then using the disjointness of $U_{2}, U_{4}, \ldots, U_{2 n}$ and (c) above we obtain a disjoint union $I_{1} \sqcup I_{2} \sqcup I_{4} \sqcup \ldots \sqcup I_{2 n}$. Note that

$$
\sum\left\{\lambda_{j}: j \in I_{1} \sqcup I_{2} \sqcup I_{4} \sqcup \ldots \sqcup I_{2 n}\right\} \leq \sum_{i=1}^{m} \lambda_{i}=1 .
$$

Then

$$
\begin{aligned}
\nu^{\prime}\left(U_{1}\right) & =\sum_{j \in I_{1}} \lambda_{j} \leq 1-\sum_{j \in I_{2}} \lambda_{j}-\sum_{j \in I_{4}} \lambda_{j}-\ldots-\sum_{j \in I_{2 n}} \lambda_{j} \\
& =1-\nu^{\prime}\left(U_{2}\right)-\nu^{\prime}\left(U_{4}\right)-\ldots-\nu^{\prime}\left(U_{2 n}\right)<1-n \alpha<\alpha
\end{aligned}
$$

by inequality $(*)$. But if $\nu^{\prime}\left(U_{1}\right)<\alpha$, then $\nu^{\prime}$ cannot belong to $W$. This is a contradiction.

CASE 2: For all $i \in\{1, \ldots, 2 n+1\}, E_{i} \neq \emptyset$. Note that we have a disjoint union $E_{1} \sqcup \ldots \sqcup E_{2 n+1}$. (If $E_{i} \cap E_{k} \neq \emptyset$ then $I_{i} \cap I_{i+1} \cap I_{k} \cap I_{k+1} \neq \emptyset$, which is impossible, since at least two of the sets $U_{i}, U_{i+1}, U_{k}, U_{k+1}$ are disjoint.) Then $\sum_{i=1}^{2 n+1} \sum_{j \in E_{i}} \lambda_{j} \leq 1$. For at least one $i$ we have $\sum_{j \in E_{i}} \lambda_{j} \leq$ $1 /(2 n+1)$. We may assume that

$$
\sum_{j \in E_{1}} \lambda_{j} \leq \frac{1}{2 n+1} .
$$


Now we have

$$
\begin{aligned}
\nu^{\prime}\left(U_{2}\right)= & \sum_{j \in I_{2}} \lambda_{j} \\
= & \sum\left\{\lambda_{j}: j \in I_{2} \cap\left(I_{1} \sqcup I_{4} \sqcup \ldots \sqcup I_{2 n}\right)\right\} \\
& +\sum\left\{\lambda_{j}: j \in I_{2} \backslash\left(I_{1} \sqcup I_{4} \sqcup \ldots \sqcup I_{2 n}\right)\right\} \\
= & \sum\left\{\lambda_{j}: j \in I_{2} \cap I_{1}\right\}+\sum\left\{\lambda_{j}: j \in I_{2} \backslash\left(I_{1} \sqcup I_{4} \sqcup \ldots \sqcup I_{2 n}\right)\right\} \\
= & \sum\left\{\lambda_{j}: j \in E_{1}\right\}+\sum\left\{\lambda_{j}: j \in I_{2} \backslash\left(I_{1} \sqcup I_{4} \sqcup \ldots \sqcup I_{2 n}\right)\right\} \\
\leq & \sum\left\{\lambda_{j}: j \in E_{1}\right\}+\sum\left\{\lambda_{j}: j \in\{1, \ldots, 2 n+1\} \backslash\left(I_{1} \sqcup I_{4} \sqcup \ldots \sqcup I_{2 n}\right)\right\} \\
\leq & \frac{1}{2 n+1}+(1-n \alpha)=\frac{2(n+1)}{2 n+1}-n \alpha<\alpha
\end{aligned}
$$

by $(* *)$. Hence, $\nu^{\prime}\left(U_{2}\right)<\alpha$, which contradicts the fact that $\nu^{\prime} \in W$.

REMARK 4.12. The previous theorem stays true if

$$
\mu=\frac{\mu_{1}+\ldots+\mu_{(n+1) l-1}}{(n+1) l-1},
$$

where $l \geq 2$ and $\mu_{i}$ 's are finitely defined by disjoint sets. The proof given is for the case $l=2$ and generalizes to arbitrary $l$.

COROLlary 4.13. There are infinitely many non-representable finitely defined topological measures on a solidly chainable $q_{0}$-space.

Proof. Non-representable extreme topological measures in Theorem 4.11 and in Remark 4.12 are also finitely defined.

\section{References}

[1] J. F. Aarnes, Quasi-states on $C^{*}$-algebras, Trans. Amer. Math. Soc. 149 (1970), 601-625.

[2] -, Quasi-states and quasi-measures, Adv. Math. 86 (1991), 41-67.

[3] -, Pure quasi-states and extremal quasi-measures, Math. Ann. 295 (1993), 575-588.

[4] - Construction of non-subadditive measures and discretization of Borel measures, Fund. Math. 147 (1995), 213-237.

[5] —, Quasi-measures on locally compact spaces, preprint.

[6] J. F. Aarnes and S. V. Butler, Super-measures and finitely defined topological measures, Acta Math. Hungar., to appear.

[7] J. F. Aarnes and A. B. Rustad, Probability and quasi-measures - a new interpretation, Math. Scand. 85 (1999), 278-284.

[8] J. P. Boardman, Quasi-measures on completely regular spaces, Rocky Mountain J. Math. 27 (1997), 447-470. 
[9] S. V. Butler, Q-functions and extreme topological measures, preprint.

[10] D. H. Fremlin, On quasi-measures, preprint.

[11] D. J. Grubb, Signed quasi-measures, Trans. Amer. Math. Soc. 349 (1997), 10811089 .

[12] —, Signed quasi-measures and dimension theory, Proc. Amer. Math. Soc. 128 (2000), 1105-1108.

[13] - , Irreducible partitions and the construction of quasi-measures, Trans. Amer. Math. Soc. 353 (2001), 2059-2072.

[14] - private communication.

[15] D. J. Grubb and T. LaBerge, Additivity of quasi-measures, Proc. Amer. Math. Soc. 126 (1998), 3007-3012.

[16] —, -, Spaces of quasi-measures, Canad. Math. Bull. 42 (1999), 291-297.

[17] F. F. Knudsen, Topology and the construction of extreme quasi-measures, Adv. Math. 120 (1996), 302-321.

[18] D. Shakhmatov, Linearity of quasi-states on commutative $C^{*}$-algebras of stable rank 1, preprint.

[19] R. F. Wheeler, Quasi-measures and dimension theory, Topology Appl. 66 (1995), $75-92$.

Department of Mathematics

University of Illinois at Urbana-Champaign

273 Altgeld Hall

1409 West Green Street

Urbana, IL 61801, U.S.A.

E-mail: svbutler@math.uiuc.edu

Received 11 June 2001;

in revised form 22 February 2002 